\title{
Critical Dispersion Distance of Silicon Nanoparticles Intercalated between Graphene Layers
}

\author{
Shuze Zhu, ${ }^{1}$ Jason Galginaitis, ${ }^{1}$ and $\mathrm{Teng}^{\mathrm{Li}}{ }^{1,2}$ \\ ${ }^{1}$ Department of Mechanical Engineering, University of Maryland, College Park, MD 20742, USA \\ ${ }^{2}$ Maryland NanoCenter, University of Maryland, College Park, MD 20742, USA \\ Correspondence should be addressed to Teng Li, lit@umd.edu
}

Received 16 October 2011; Accepted 25 January 2012

Academic Editor: Sulin Zhang

Copyright ( 2012 Shuze Zhu et al. This is an open access article distributed under the Creative Commons Attribution License, which permits unrestricted use, distribution, and reproduction in any medium, provided the original work is properly cited.

\begin{abstract}
Nanocomposites of silicon nanoparticles ( $\mathrm{Si}$ NPs) dispersed in between graphene layers emerge as potential anode materials of high-charge capacity for lithium-ion batteries. A key design requirement is to keep Si NPs dispersed without aggregation. Experimental design of the Si NP dispersion in graphene layers has remained largely empirical. Through extensive molecular dynamics simulations, we determine a critical NP dispersion distance as the function of NP size, below which Si NPs in between graphene layers evolve to bundle together. These results offer crucial and quantitative guidance for designing NP-graphene nanocomposite anode materials with high charge capacity.
\end{abstract}

Rechargeable lithium-ion batteries with high energy capacity and long cycle life are in great demand for applications such as portable devices and electric vehicles [1-3]. Silicon has the highest known theoretical charge capacity, more than ten times higher than the existing graphite anodes [4]. However, the huge volume changes (up to 400\%) of silicon upon charging/discharging cause its pulverization and capacity fading, posing a significant challenge to use silicon as anodes $[4,5]$. Nanocomposites of silicon nanoparticles (Si NPs) dispersed in between graphene layers are emerging as a novel and promising solution to high-performance Si-based anode materials $[6,7]$. Nanoscale silicon materials (e.g., nanoparticles and nanowires) have been shown to be able to sustain much larger volume change without pulverization than their bulk counterpart $[6,8]$. The graphene layers of high mechanical flexibility and large surface area serve as a percolating scaffold network to help accommodate the volume change of the Si NPs during charging/discharging and ensure facile electron transport. A key design requirement of such nanocomposite anode materials is to keep the Si NPs evenly dispersed in between the graphene layers [6,9]. Given their huge surface area over volume ratio, Si NPs that are too close to each other tend to bundle together to reduce surface energy. The aggregations of the Si NPs become more prone to pulverization than dispersed Si NPs. On the other hand, the charging capacity of the nanocomposite anode material scales with the total volume of the Si NPs that can be dispersed in between the graphene layers. Therefore, the optimal design of Si NP-graphene-based anode materials hinges upon determining the maximum dispersion density of the Si NPs in the graphene layers without Si NP aggregation. Such a critical dispersion of the Si NPs in the graphene layers remains elusive so far, as most experimental studies in this regard are largely empirical $[6,7]$. To address such a crucial but large unexplored issue, in this paper we conduct extensive molecular dynamics simulations to determine a critical dispersion distance of the Si NPs as the function of NP size, below which Si NPs in between two graphene layers evolve to bundle together.

The dispersion of the Si NPs in between graphene layers is governed by the energetic interplay between the graphene layers and the Si NPs [10]. The typical size of the Si NPs dispersed in between graphene layers is a few nanometers in diameter, much larger than the equilibrium graphenegraphene interlayer distance $(\sim 0.34 \mathrm{~nm})$. As a result, the graphene layers are locally separated by the Si NPs and assume corrugated morphology to wrap around the $\mathrm{Si}$ NPs [6]. Such corrugated morphology of the graphene layers results from the following energetic interplay. On the one hand, the graphene-graphene interlayer interaction is 

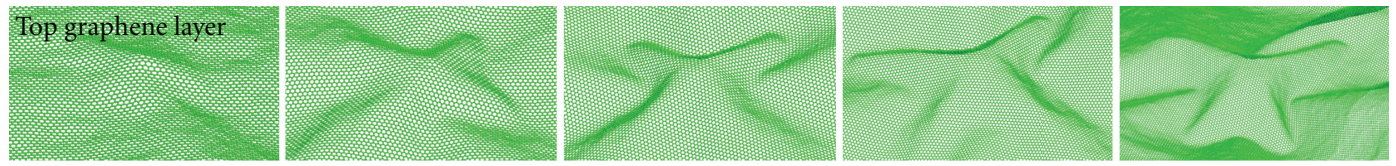

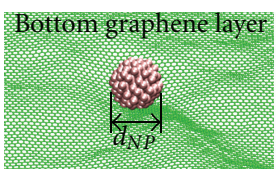

$d_{N P}=2 \mathrm{~nm}$
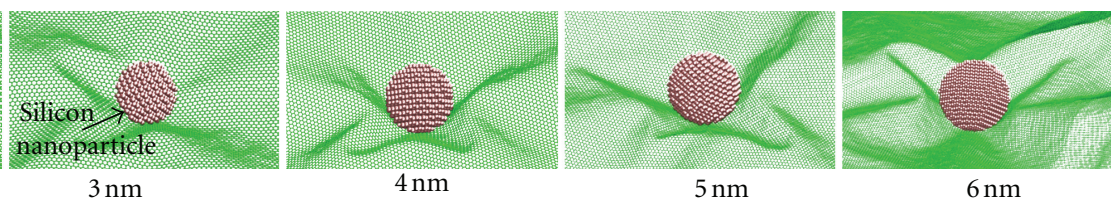

FIGURE 1: The morphology of graphene with the intercalation of a single Si NP of various diameters, $d_{\mathrm{NP}}$. For visual clarity, the top panel shows the top graphene layer, and the bottom one shows the Si NP and the bottom graphene layer (only the portion near the Si NP is shown). Note the ridged morphology formation as $d_{\mathrm{NP}}$ increases.

mainly van der Waals type. Therefore, the graphene layers tend to assume their equilibrium distance to reduce the interaction energy. On the other hand, the corrugation of graphene layers due to Si NP intercalation causes the bending and stretching of the graphene, and thus leads to an increase of the graphene strain energy. The tighter the graphene layers wrap around the Si NPs, the higher the resulting graphene strain energy, and the lower the graphenegraphene interaction energy. The equilibrium morphology of the graphene-NP structure is dictated by minimizing the total free energy of the structure, which includes two dominant contributions, that is, the graphene strain energy and the graphene-graphene interaction energy. Given the large in-plane dimension of the graphene (on the order of microns or higher), the weak interaction between the graphene and Si NPs (also van der Waals type) is of secondary significance in the total free energy. So is the strain energy of the Si NPs, which is negligible due to the weak graphene-Si interaction and out-of-plane flexibility of the graphene.

We first conduct molecular dynamics (MD) simulations to determine the equilibrium morphology of the graphene layers with a single Si NP intercalated in between, which corresponds to the limiting case when the Si NPs are widely dispersed in between graphene layers, thus the interaction among Si NPs is negligible. In consideration of the computational cost, we model a graphene bilayer with a Si NP intercalated in between, corresponding to the two closest neighbor graphene layers that wrap around the Si NP in a real material. Such a simplified model can capture the dominant energetic interplay, given that the graphene-graphene interlayer interaction decays quickly as their distance increases, and thus the contribution from the next neighbor graphene layers to the interaction energy is of secondary significance.

In the MD simulations, each graphene layer has a size of $50 \mathrm{~nm}$ by $70 \mathrm{~nm}$. Single-crystal Si NPs of various diameters (i.e., 2 6 $\mathrm{nm}$ ) are used to study the effect of NP size on the corrugated graphene morphology. The carbon-carbon (CC) covalent bonds in the graphene are described by the adaptive intermolecular reactive bond order (AIREBO) potential [11]. The nonbonded graphene-graphene interlayer interaction and the graphene-Si interaction are described by two Lennard-Jones pair potentials [12], $V_{\mathrm{C}-\mathrm{C}}(r)=$ $4 \varepsilon_{\mathrm{C}-\mathrm{C}}\left(\sigma_{\mathrm{C}-\mathrm{C}}^{12} / r^{12}-\sigma_{\mathrm{C}-\mathrm{C}}^{12} / r^{6}\right)$ and $V_{\mathrm{C}-\mathrm{Si}}(r)=4 \varepsilon_{\mathrm{C}-\mathrm{Si}}\left(\sigma_{\mathrm{C}-\mathrm{Si}}^{12} / r^{12}-\right.$ $\left.\sigma_{\mathrm{C}-\mathrm{Si}}^{12} / r^{6}\right)$, respectively, where $\varepsilon_{\mathrm{C}-\mathrm{C}}=0.00284 \mathrm{eV}, \sigma_{\mathrm{C}-\mathrm{C}}=$ $0.34 \mathrm{~nm}, \varepsilon_{\mathrm{C}-\mathrm{Si}}=0.00213 \mathrm{eV}$, and $\sigma_{\mathrm{C}-\mathrm{Si}}=0.15 \mathrm{~nm}$. To reduce the computation cost, the Si NP is assumed to be rigid (i.e., whose bonding energy remains as a constant). In each MD simulation case, the graphene bilayer, with free boundary conditions for both layers, is prescribed with an initial morphology that, near the Si NP, it bulges out into a conical dome in each layer to house the Si NP inside and far away from the Si NP, it remains flat with an interlayer distance of $0.6 \mathrm{~nm}$. The Si NP is first fixed, and the graphene bilayer is equilibrated for 30 ps to minimize the dependence of the final results on the initial prescribed morphology, then the Si NP is set free to evolve with the graphene bilayer until an equilibrium is reached. The MD simulations are carried out using LAMMPS with Canonical Ensemble at temperature $300 \mathrm{~K}$ and with time step $1 \mathrm{fs}$.

Figure 1 shows the simulated morphology of graphene with a single Si NP intercalated in between of various diameters, $d_{\mathrm{NP}}=2 \mathrm{~nm}, 3 \mathrm{~nm}, 4 \mathrm{~nm}, 5 \mathrm{~nm}$, and $6 \mathrm{~nm}$, respectively. For visual clarity, the top panel in Figure 1 shows the top graphene layer and the bottom one shows the Si nanoparticle and the bottom graphene layer. If the Si NP is small (e.g., $d_{\mathrm{NP}}=2 \mathrm{~nm}$ ), each graphene layer bulges out into a dome to wrap around the Si NP. As the size of the Si NP increases, the graphene layers start to form ridged morphology to wrap around the Si NP. The locations of such ridges are approximately complementary in the top and bottom graphene layers. The formation of ridged morphology in the graphene bilayer can be understood as follows. As the size of the Si NP increases, forming a smooth conical dome in each graphene layer to wrap around the Si NP requires accommodating the out-of-plane deflection of the graphene bilayer through the in-plane stretching in radial direction and compressing in hoop direction of the graphene bilayer. Such in-plane deformation of the graphene is energetically unfavorable, given graphene's ultrahigh inplane stiffness. By contrast, given the much amenable outof-plane flexibility of graphene, accommodating the out-ofplane deflection of the graphene bilayer by bending each graphene layer to form ridges corresponds to a lower total free energy state, thus is more favorable. 

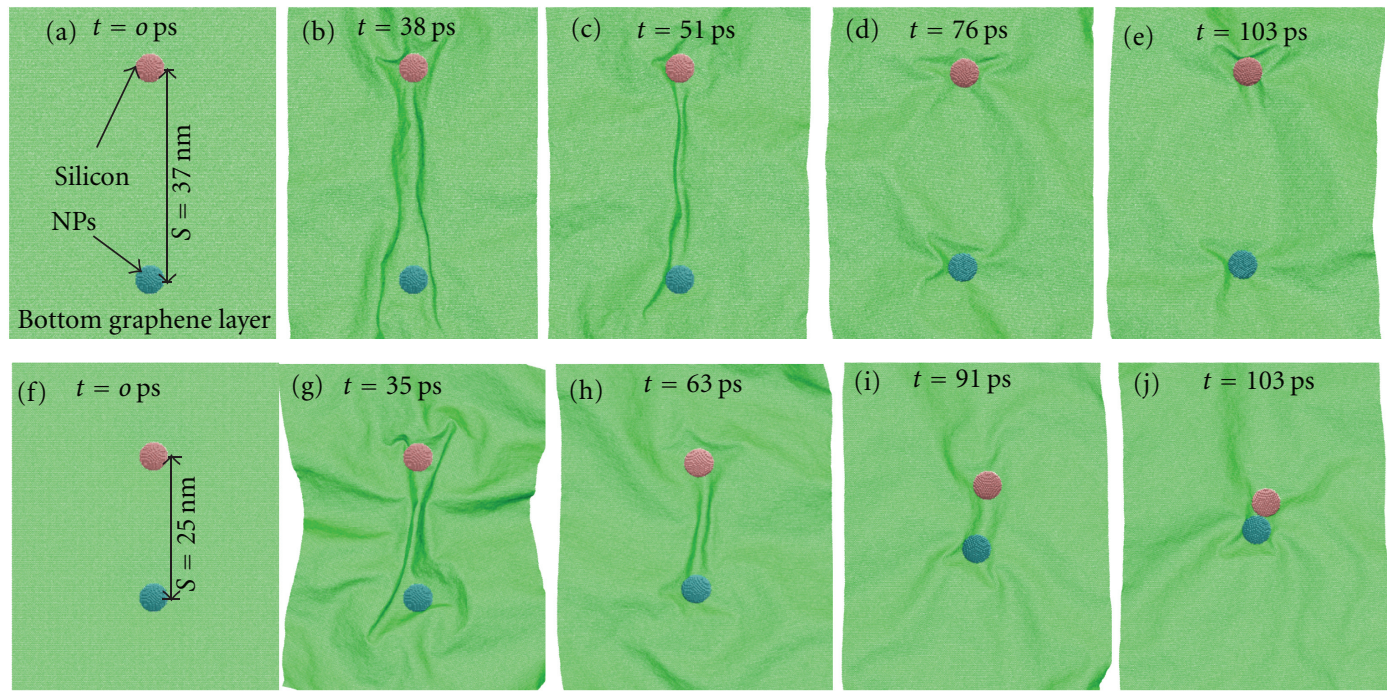

FIgURE 2: (a)-(e) the morphologic evolution of the Si NP-graphene system over simulation time. For visual clarity, only the bottom graphene layer and the Si NPs are shown. When the distance between the two Si NPs is sufficiently large (here $d_{\mathrm{NP}}=5 \mathrm{~nm}$, and $S=37 \mathrm{~nm}$ ), the two Si NPs remain dispersed and are wrapped individually by the graphene bilayer. The corrugated graphene morphology near each Si NP is similar to that shown in Figure 1. (f)-(j) when the distance between the two Si NPs is below a critical value (here $d_{\mathrm{NP}}=5 \mathrm{~nm}$, and $S=25 \mathrm{~nm}$ ), the two Si NPs evolve to come closer and eventually bundle together. (Supplemental materials: two videos showing the morphologic evolution of the Si NP-graphene system corresponding to (a)-(e) and (f)-(j) are available at http://ter.ps/dispersed and http://ter.ps/bundled, resp., available online at doi: 10.1155/2012/375289).

We next consider how the structural morphology of the Si NP-graphene nanocomposites evolves as the dispersion distance between the Si NPs varies. To capture the dominant underlying physics of such a morphologic evolution within reasonable computation cost, we model a graphene bilayer with two neighboring Si NPs intercalated in between. Similar materials parameters and numerical strategy as those described above are used in the MD simulations. For a given Si NP size, MD simulations are conducted over a range of Si NP dispersion distance. Two modes of morphologic evolution emerge from the simulations, as illustrated in Figure 2. If the dispersion distance between $\mathrm{Si}$ NPs, denoted by $S$, is sufficiently large (e.g., $S=37 \mathrm{~nm}$ for $d_{\mathrm{NP}}=5 \mathrm{~nm}$ ), the graphene bilayer in between two neighboring Si NPs is first corrugated to form a long tunnel (Figure 2(b)). The tunnel formation, however, results in increased strain energy in the graphene, therefore is energetically unfavorable. As a result, the long tunnel in the graphene bilayer evolves to disappear, preventing the two Si NPs to migrate toward each other (Figures 2(c) and 2(d)). At equilibrium, the Si NPs remain dispersed and are individually wrapped by the graphene (Figure 2(e)). The corrugated morphology of the graphene near each Si NP is similar to that depicted in Figure 1. If the dispersion distance between the Si NPs is small (e.g., $S$ $=25 \mathrm{~nm}$ for $d_{\mathrm{NP}}=5 \mathrm{~nm}$ ), the corrugated graphene bilayer between the two neighboring Si NPs can form a short tunnel, which facilitates the migration of the two Si NPs toward each other. As the two Si NPs evolve to come closer, the length of the tunnel becomes shorter (e.g., Figures 2(h) and 2(i)), resulting in a decrease of the strain energy of the graphene, which is thus energetically favorable. Eventually, the two Si NPs evolve to form a bundle, which is wrapped together by the graphene bilayer (Figure 2(j)).

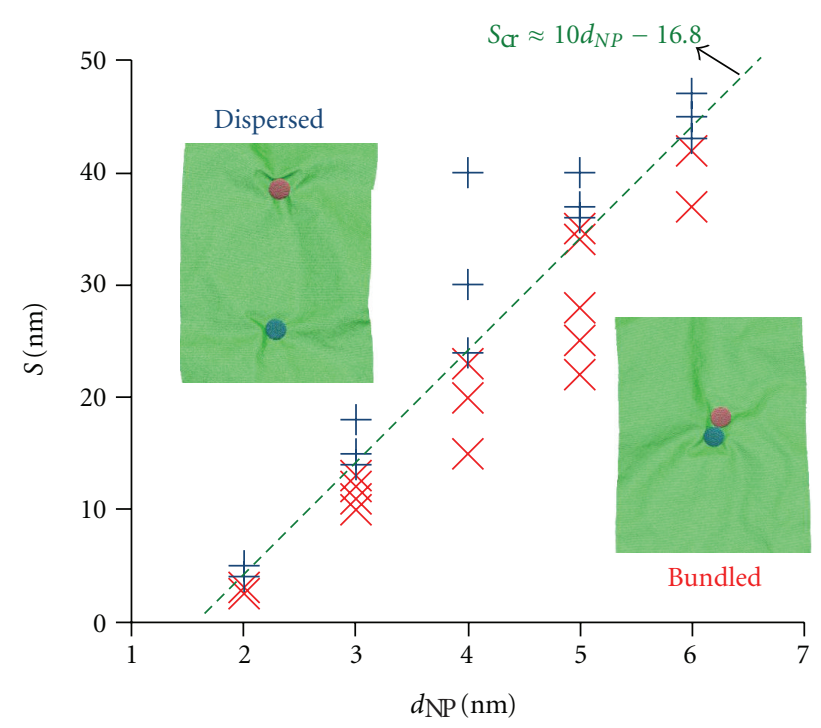

FIGURE 3: A phase diagram of the morphologic evolution of the $\mathrm{Si}$ NP-graphene system in the space of NP distance and diameter. "+" denote the MD simulation cases in which Si NPs remain dispersed and " $X$ " denote those in which Si NPs evolve to bundle together. A critical dispersion distance of the Si NPs, $S_{\mathrm{cr}}$, can be determined, which has an approximately linear dependence on $d_{\mathrm{NP}}$.

Also emerging from extensive MD simulations is a critical dispersion distance of Si NPs, $S_{\mathrm{cr}}$, above which the Si NPs remain dispersed and below which the Si NPs evolve to bundle together. Figure 3 plots $S_{\mathrm{cr}}$ as a function of the diameter of the Si NP, $d_{\mathrm{NP}}$. There is an approximately linear dependence of $S_{\mathrm{cr}}$ on $d_{\mathrm{NP}}$, that is, $S_{\mathrm{cr}} \approx 10 d_{\mathrm{NP}}-16.8$. Results in Figure 3 can serve as guidance for the material 
and structural design of Si NP-graphene nanocomposites as anode materials for lithium-ion batteries. For example, for a given design criteria of charging capacity, the maximum volume expansion of the Si NPs upon lithiation can be estimated. The corresponding enlarged size of the Si NPs, instead of the size of pristine Si NPs, should be used to determine a critical dispersion distance to prevent the aggregation of the Si NPs.

In summary, we conduct extensive MD simulations to investigate the morphologic evolution of Si NPs intercalated in between graphene layers, from which a critical dispersion distance of the Si NPs can be determined below which neighboring Si NPs evolve to form a bundle. A roughly linear dependence of the critical distance of the Si NPs on its size is revealed. The ridged morphology of graphene has been observed in recent experiments of substrate-supported graphene with NPs intercalated between the graphene and the substrate [13]. It is further shown that tunneling ridges form between NPs below a critical dispersion distance of the NPs, though the rigid substrate to some extent inhibits the neighboring NPs evolving to bundle together. In our MD simulations, we consider the interaction between two closest neighbor NPs of same size intercalated between graphene layers. In real materials, the variation of the NP sizes and the randomness of the NP distribution render more complex morphologic features that pose significant challenge to be characterized solely by modeling. Nonetheless, results from the present study captures the dominant underlying energetics of NP intercalated in between graphene layers. Furthermore, since the graphene-NP interaction is of secondary importance in determining the corrugated graphene morphology, results from the present study are generally applicable to graphene layers with other anode materials in NP form (e.g., $\mathrm{SnO}_{2}$ and $\mathrm{TiO}_{2}$ ) intercalated in between [14-16]. While the mechanistic understanding and quantitative determination of the critical dispersion distance of Si NPs intercalated between graphene layers shed crucial light on optimizing the design of Si NP-graphene nanocomposites as the highperformance anode materials in lithium-ion batteries, systematic experimental explorations are desirable to validate and further leverage the full potential of the present study.

\section{Acknowledgments}

This paper is supported by National Science Foundation (Grants no. 1069076 and no. 1129826) and a UMD GRB Summer Research Award. The authors acknowledge the constructive comments from anonymous reviewers.

\section{References}

[1] M. S. Whittingham, "Lithium batteries and cathode materials," Chemical Reviews, vol. 104, no. 10, pp. 4271-4301, 2004.

[2] K. Kang, Y. S. Meng, J. Bréger, C. P. Grey, and G. Ceder, "Electrodes with high power and high capacity for rechargeable lithium batteries," Science, vol. 311, no. 5763, pp. 977-980, 2006.

[3] J. M. Tarascon and M. Armand, "Issues and challenges facing rechargeable lithium batteries," Nature, vol. 414, no. 6861, pp. 359-367, 2001.
[4] B. A. Boukamp, G. C. Lesh, and R. A. Huggins, "All-solid lithium electrodes with mixed-conductor matrix," Journal of the Electrochemical Society, vol. 128, no. 4, pp. 725-729, 1981.

[5] U. Kasavajjula, C. Wang, and A. J. Appleby, "Nano- and bulk-silicon-based insertion anodes for lithium-ion secondary cells," Journal of Power Sources, vol. 163, no. 2, pp. 1003-1039, 2007.

[6] J. K. Lee, K. B. Smith, C. M. Hayner, and H. H. Kung, "Silicon nanoparticles-graphene paper composites for Li ion battery anodes," Chemical Communications, vol. 46, no. 12, pp. 20252027, 2010.

[7] J.-Z. Wang, C. Zhong, S.-L. Chou, and H.-K. Liu, "Flexible free-standing graphene-silicon composite film for lithium-ion batteries," Electrochemistry Communications, vol. 12, no. 11, pp. 1467-1470, 2010.

[8] C. K. Chan, H. Peng, G. Liu et al., "High-performance lithium battery anodes using silicon nanowires," Nature Nanotechnology, vol. 3, no. 1, pp. 31-35, 2008.

[9] Z. Y. Wang, H. Zhang, N. Li, Z. J. Shi, Z. N. Gu, and G. P. Cao, "Laterally confined graphene nanosheets and graphene/ $\mathrm{SnO}_{2}$ composites as high-rate anode materials for lithium-ion batteries," Nano Research, vol. 3, no. 10, pp. 748756, 2010.

[10] T. Li, "Extrinsic morphology of graphene," Modelling and Simulation in Materials Science and Engineering, vol. 19, no. 5, Article ID 054005, 2011.

[11] D. W. Brenner, O. A. Shenderova, J. A. Harrison, S. J. Stuart, B. Ni, and S. B. Sinnott, "A second-generation reactive empirical bond order (REBO) potential energy expression for hydrocarbons," Journal of Physics Condensed Matter, vol. 14, no. 4, pp. 783-802, 2002.

[12] S. J. Stuart, A. B. Tutein, and J. A. Harrison, "A reactive potential for hydrocarbons with intermolecular interactions," Journal of Chemical Physics, vol. 112, no. 14, pp. 6472-6486, 2000.

[13] M. Yamamoto, W. G. Cullen, and M. S. Fuhrer, Private communication.

[14] H. Kim, S.-W. Kim, Y.-U. Park et al., " $\mathrm{SnO}_{2}$ /graphene composite with high lithium storage capability for lithium rechargeable batteries," Nano Research, vol. 3, no. 11, pp. 813$821,2010$.

[15] S. M. Paek, E. Yoo, I. Honma et al., "Enhanced cyclic performance and lithium storage capacity of $\mathrm{SnO}_{2}$ /graphene nanoporous electrodes with three-dimensionally delaminated flexible structure," Nano Letters, vol. 9, no. 1, pp. 72-75, 2009.

[16] D. Wang, D. Choi, J. Li et al., "Self-assembled $\mathrm{TiO}_{2}$-graphene hybrid nanostructures for enhanced Li-ion insertion," ACS Nano, vol. 3, no. 4, pp. 907-914, 2009. 

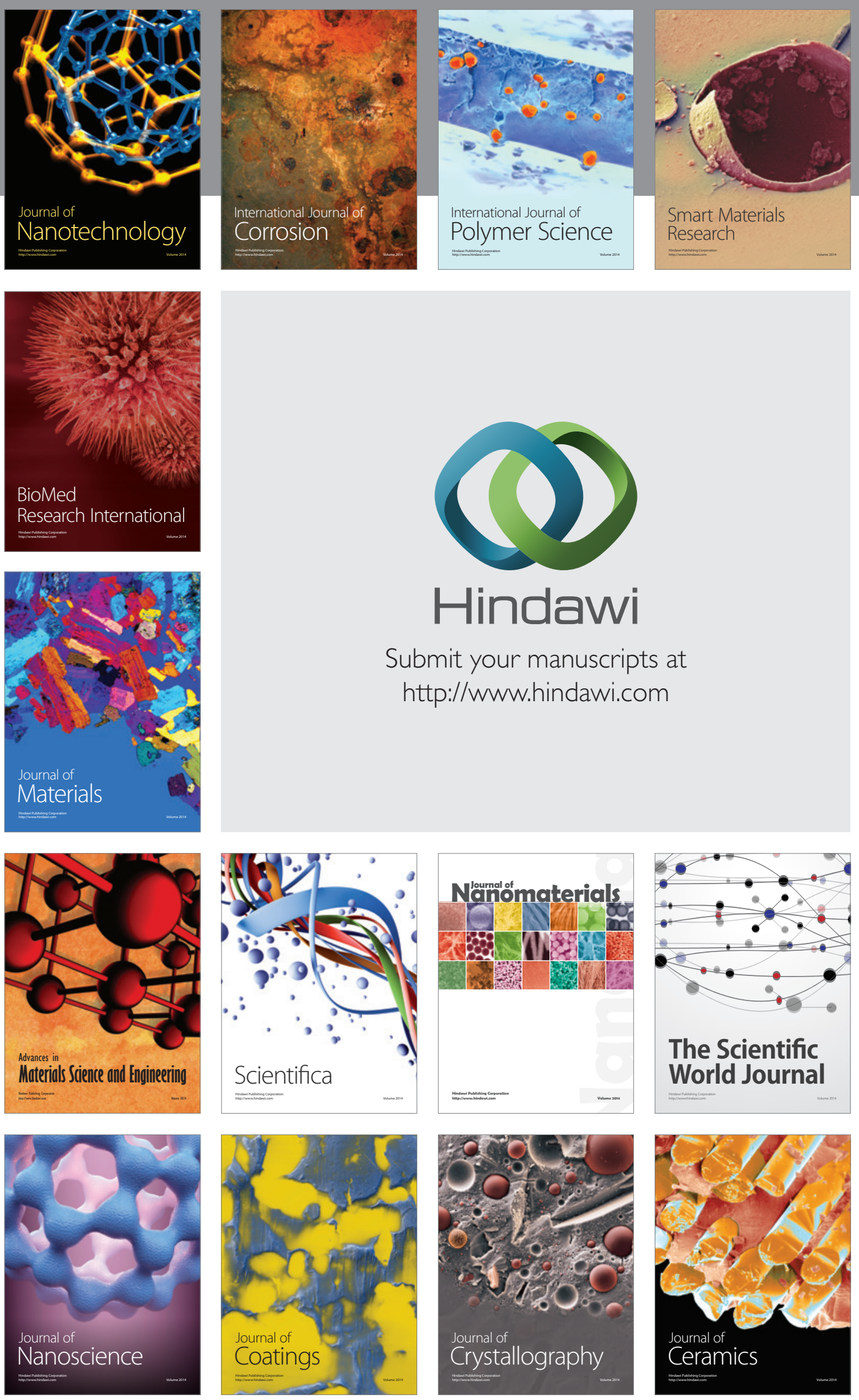

The Scientific World Journal

Submit your manuscripts at

http://www.hindawi.com

\section{World Journal}

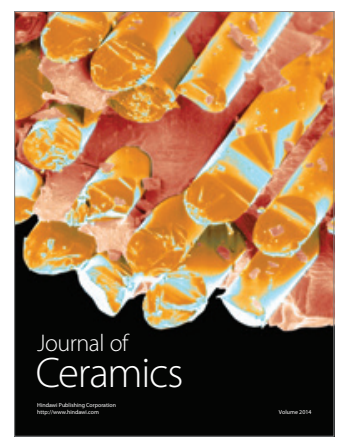

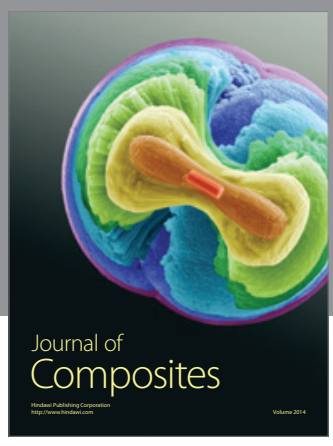
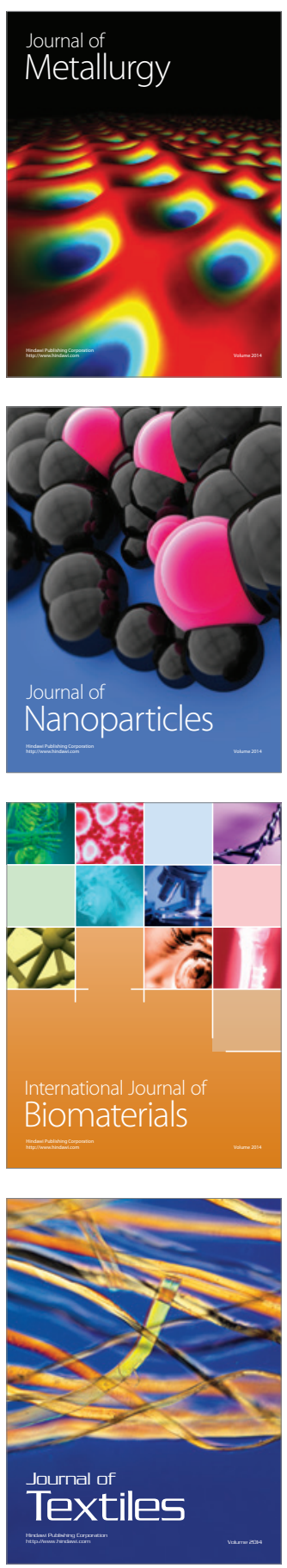